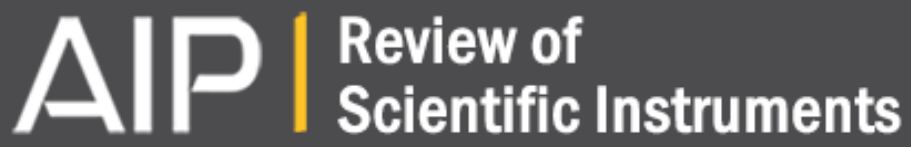

\section{A serial-kinematic nanopositioner for high-speed atomic force microscopy}

Sachin P. Wadikhaye, Yuen Kuan Yong, and S. O. Reza Moheimani

Citation: Review of Scientific Instruments 85, 105104 (2014); doi: 10.1063/1.4897483

View online: http://dx.doi.org/10.1063/1.4897483

View Table of Contents: http://scitation.aip.org/content/aip/journal/rsi/85/10?ver=pdfcov

Published by the AIP Publishing

\section{Articles you may be interested in}

High-stroke silicon-on-insulator MEMS nanopositioner: Control design for non-raster scan atomic force microscopy

Rev. Sci. Instrum. 86, 023705 (2015); 10.1063/1.4907908

Diagonal control design for atomic force microscope piezoelectric tube nanopositioners

Rev. Sci. Instrum. 84, 023705 (2013); 10.1063/1.4790474

Invited Review Article: High-speed flexure-guided nanopositioning: Mechanical design and control issues Rev. Sci. Instrum. 83, 121101 (2012); 10.1063/1.4765048

Local raster scanning for high-speed imaging of biopolymers in atomic force microscopy

Rev. Sci. Instrum. 82, 063703 (2011); 10.1063/1.3600558

Enlarged atomic force microscopy scanning scope: Novel sample-holder device with millimeter range

Rev. Sci. Instrum. 78, 095107 (2007); 10.1063/1.2773623

Frustrated by

old technology?

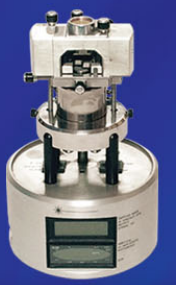

Is your AFM dead and can't be repaired?

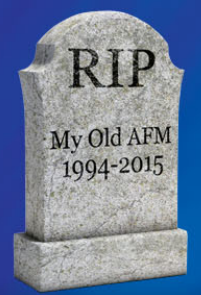

Sick of bad customer support?

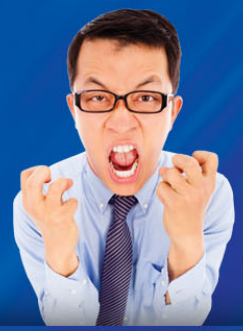

It is time to upgrade your AFM

Minimum \$20,000 trade-in discount for purchases before August 31st

Asylum Research is today's technology leader in AFM

dropmyoldAFM@oxinst.com

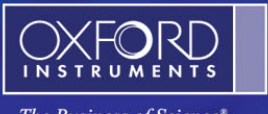




\title{
A serial-kinematic nanopositioner for high-speed atomic force microscopy
}

\author{
Sachin P. Wadikhaye, ${ }^{\text {a) }}$ Yuen Kuan Yong, and S. O. Reza Moheimani \\ School of Electrical Engineering and Computer Science, The University of Newcastle, Callaghan, NSW, \\ Australia
}

(Received 25 July 2014; accepted 28 September 2014; published online 13 October 2014)

\begin{abstract}
A flexure-guided serial-kinematic XYZ nanopositioner for high-speed Atomic Force Microscopy is presented in this paper. Two aspects influencing the performance of serial-kinematic nanopositioners are studied in this work. First, mass reduction by using tapered flexures is proposed to increased the natural frequency of the nanopositioner. $25 \%$ increase in the natural frequency is achieved due to reduced mass with tapered flexures. Second, a study of possible sensor positioning in a serial-kinematic nanopositioner is presented. An arrangement of sensors for exact estimation of cross-coupling is incorporated in the proposed design. A feedforward control strategy based on phaser approach is presented to mitigate the dynamics and nonlinearity in the system. Limitations in design approach and control strategy are discussed in the Conclusion. () 2014 AIP Publishing LLC. [http://dx.doi.org/10.1063/1.4897483]
\end{abstract}

\section{INTRODUCTION}

Development of nanopositioning systems has been at the core of the research aimed at achieving higher throughput in scanning probe microscopy ${ }^{1,2}$ high precision optical positioning, ${ }^{3}$ and nano manufacturing. ${ }^{4}$ The drive for achieving improved performance steers the design of these high-precision instruments towards satisfying stringent specifications such as range and bandwidth. For example, flexureguided nanopositioners driven by piezoelectric stack actuators have shown to attain high-speed for critical applications such as video rate Atomic Force Microscopy (AFM) ${ }^{5-9}$ The capability of flexure based nanopositioner in the AFM to move the sample at video rate is an important development for imaging biological samples. This has enabled the researchers to use the instrument to record the dynamic behavior of biological processes. ${ }^{5}$ In this article, the design, characterization, and use of a high-speed serial-kinematic XYZ nanopositioner in a high-speed AFM are presented. The device incorporates a novel flexure design and appropriate sensor positioning in an attempt to make the nanopositioner fast and accurate.

Higher imaging rates can be achieved by developing a high bandwidth XYZ nanopositioner that can move the sample at higher speeds. However, the design constraints limit the achievable bandwidth in such nanopositioners. For instance, there is a trade off between achievable natural frequency and the travel range of the nanopositioning stage. ${ }^{1,2}$ This design limitation restricts the achievable range of the stage if stiffness of flexures is increased. Designs such as that presented by Schitter et al., ${ }^{7}$ used two piezoelectric actuators on either side of the stage to increase the overall stiffness of the mechanism without much affecting the range. Also, in several designs, ${ }^{8,10}$ analytical stiffness calculations were used to optimise the stiffness of the flexures of the stage. An extensive review of flexure based nanopositioning systems is presented by Yong et al., ${ }^{2}$ which suggests a trend line between

a)E-mail: sachin.wadikhaye@uon.edu.au achievable natural frequency and range of the stages. Design of nanopositioners that can achieve performance better than this trend line is a challenging task.

The mass of the structure plays a very significant role in the design of nanopositioners. In most of the high-speed nanopositioning stages reported in literature, the mass is often concentrated on the central stage on which the sample is mounted. ${ }^{5,6,9-13}$ However, mass reduction to achieve a higher natural frequency is often overlooked. In a recent article, ${ }^{14}$ mass reduction is achieved by the removal of some material of the stage at low stress points. This paper explores parameters that would affect the mass and stiffness of the nanopositioning stage. The aim of this study is to achieve high natural frequencies in fast serial-kinematic nanopositioners. In particular, we propose the use of tapered flexures in the nanopositioner design. To the best of author's knowledge, this has never been attempted in the past. Parametric studies presented here indicate that tapering can be used to modify the height of the stage and thereby reduce the mass. Design examples of fast serialkinematic nanopositioners using tapered and straight flexures are presented and compared. The stage with tapered flexures is observed to have $25 \%$ higher natural frequency than the stage with straight flexures.

Displacement measurement in a serial-kinematic nanopositioner is not straightforward. The sensor positioning is hard to comprehend due to interlacing of stages. In most of serial-kinematic nanopositioners reported in the literature, the images are generated using the displacement of stages instead of the displacement of the sample. ${ }^{5,6,8,10}$ Also, in some nanopositioners sensors are mounted at non-optimum positions which generally neglect the cross talks from other axes. ${ }^{8}$ Measurements obtained using such arrangement may adversely affect the accuracy of the images produced by the AFM. In this paper, brief account of possible configurations in which sensors can be mounted on serial-kinematic nanopositioners is analyzed. The best configuration is suggested which measures the displacement of the sample and not that of the individual stages. Such an arrangement estimates 
the lateral position with respect to a fixed structure which eventually helps in measuring the exact cross-coupling between stages as seen by the sample.

Apart from the mechanical design, the performance of the nanopositioner can be hampered by operational limitations such as system dynamics or actuator behavior. ${ }^{1,6,15} \mathrm{Sev}-$ eral control techniques to overcome vibrations and nonlinear behavior to improve the scanning speeds of AFM have been proposed by researchers over the past decade. High performance controllers such as positive position feedback (PPF), ${ }^{16}$ integral resonant control (IRC), ${ }^{17,18}$ loop shaping using $H_{\infty},{ }^{11}$ LQG controllers, ${ }^{19}$ and several others have been proposed. Such controllers are either computationally intensive or have a high implementation cost which may carry the cost of dedicated hardware such as FPGA or FPAA. ${ }^{8,9}$ A cost effective approach to mitigate the effects of vibration and nonlinearity is to use the feedforward control techniques. ${ }^{20}$ Most feedforward techniques involve inversion of the linear dynamic model and the hysteresis model of the plant. Not to mention, hysteresis modeling for feedforward control itself has been a subject of intense research in the last few decades. Modeling techniques such as Preisach model, ${ }^{21}$ Prandtl-Ishlinskii model, ${ }^{22}$ and Maxwell resistive capacitor (MRC) ${ }^{23}$ have been reported in the literature. However, such approaches are demanding in terms of modeling complexity and parameter sensitivity. In this paper, a phaser based approach ${ }^{24}$ is used for hysteresis modeling. This technique eliminates the stringent parameter dependency while sufficiently suppressing the hysteretic effects.

The remainder of the paper is organized in the following manner. In Sec. II, a parametric study of straight and tapered flexures is presented. An analytical framework for stiffness calculations of tapered flexures is laid down and the effect of each parameter on the stiffness of the stage is evaluated. A comparison of the designs based on straight and tapered flexures is presented. Section III presents the study of possible sensor positions for cross-coupling measurements. In Sec. IV, the design of proposed nanopositioner is discussed in detail. Section $\mathrm{V}$ presents characterization of the nanopositioner and explains the control strategy. Imaging and tracking performance are presented and discussed in Sec. VI. Finally, Sec. VII concludes the paper.

\section{PARAMETRIC STUDY OF TAPERED FLEXURES}

For a system such as that shown in Fig. 1, whose dynamics resemble that of a damped harmonic oscillator, high resonance frequency can be achieved by increasing the stiffness and by reducing the mass of the system. There has been studies presented in the literature to analytically or numerically estimate and decide the stiffness of the flexures such that a required structural stiffness is achieved for a high speed nanopositioner design. However, the designer must strike a compromise between natural frequency and travel range. The travel range of a piezoelectric actuator is limited by the stiffness of structure, conversely, to achieve higher natural frequency, hence higher scanning speed, the structure should be made stiffer. This bottleneck in design of a flexure guided nanopositioners has been acknowledged several times

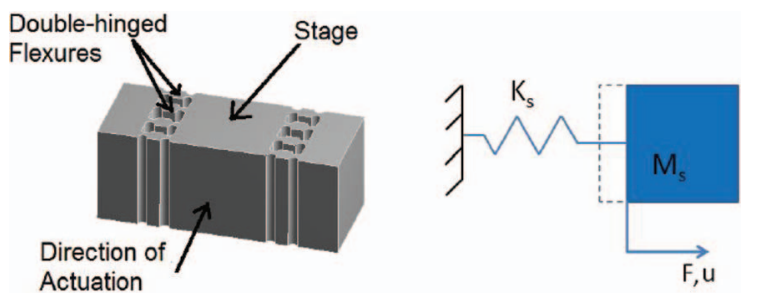

FIG. 1. Construction and modeling of a nanopositioning stage.

in the literature. ${ }^{2,8,10}$ Mass reduction has often been attempted based on the stress distribution estimates from finite element analysis. ${ }^{14}$ In a monolithic structure as shown in Fig. 1, the flexure dimensions often influence the stage size. To the authors' knowledge, the flexure used in all the designs reported in literature have constant height. The height of the flexures is a critical parameter in achieving high out of plane stiffness in flexures. Therefore, the stage height is kept consistent with the flexure height. However, mass reduction may result in improvements in the performance of nanopositioning system explained as follows:

1. The natural frequency of the system is inversely proportional to the mass of the system. Therefore, reduction in mass may result in enhanced natural frequency.

2. Mass reduction of the nanopositioning stage may result in higher out-of-plane stiffness to mass ratios compared to that in actuation direction. This ensures a dominant first resonance along the actuation direction while shifting the out-of-plane modes higher in the frequency spectrum..$^{8}$

3. For a second order system, the damping ratio is inversely proportional to the mass given by the relation $\zeta=c / 2 \sqrt{m k} .^{25}$ Thus, systems with a higher mass result in taller and sharper resonance peaks. This in turn leads to lower gain margins for tracking control which is critical for high speed nanopositioning systems. Reducing the mass may increase the damping ratio and thereby relaxing the gain margins.

Tapering of flexures can be used to reduce the height and hence the mass of the central stage. A higher natural frequency can thus be achieved by the reduction of mass. However, the two hinges in the doublehinged flexure form a couple giving constrained motion by an equal bending motion. Tapering will decrease the bending stiffness of the hinge at the tapered side as the height is reduced. The unequal bending stiffness will induce unwanted stresses in the flexure. One way to avoid these stresses being developed in the tapered flexure is to match the bending stiffness of the two hinges. If the minimum thickness $t$ is kept constant, an identical stiffness can be achieved for both hinges so that $\frac{K_{y y 0}}{K_{y y T}}=1$. To achieve this, the ratio between radii of the two hinges in a tapered flexure should be 26

$$
\frac{H_{0}}{H_{T}}=\left(\frac{R_{T}}{R_{0}}\right)^{3} .
$$

To calculate the stiffness of flexures, consider the strain energy equation for bending of a generic rectangular beam 
flexure as

$$
U=\int_{0}^{L} \frac{M^{2} d x}{2 E I(x)}+\int_{0}^{L} \frac{\alpha V d x}{2 G A(x)},
$$

where $U$ is the strain energy in bending, $L$ is the length of the flexure, $E$ is the modulus of elasticity of the material, $M$ and $V$ are moment and shear force acting on the flexures, $A(x)=H$ $\times t(x)$ represents variation of cross-section of the flexures along the length, and $I(x)=\frac{H \times t(x)^{3}}{12}$ is the moment of inertia. $^{27}$

The stiffness of tapered flexures can be calculated by modifying (2) to allow $H$ to be a function of $\mathrm{x}$, i.e., $H=H(x)$, which implies, $A(x)=H(x) \times t(x)$ and $I(x)=\frac{H(x) \times t(x)^{3}}{12}$. The variation of height $H(x)$ along the length of the flexure is given by

$$
H(x)=H_{0}-S l \times x,
$$

where $S l=\left(H_{0}-H_{T}\right) / L$ is the slope of the tapered side as shown in Fig. 2.

When considering the calculation of stiffness of doublehinged flexures the cross-sectional area and second moment of inertia should be considered as functions of thickness. The variation in the thickness of the double-hinged tapered flexure along the length can be written as

$$
t(x)= \begin{cases}t+2\left[R_{0}-\sqrt{x\left(2 R_{0}-x\right)}\right] & \text { if } x \in[0, m], \\ t+2\left[R_{0}-\sqrt{(x)\left(2 R_{0}-x\right)}\right] & \text { if } x \in[m, n], \\ t+2 R_{T} & \text { if } x \in[n, o], \\ t+2\left[R_{T}-\sqrt{(k)\left(2 R_{T}-k\right)}\right] & \text { if } x \in[o, p], \\ t+2\left[R_{T}-\sqrt{(g)\left(2 R_{T}-g\right)}\right] & \text { if } x \in[p, L],\end{cases}
$$

where $m=R_{0}, n=2 R_{0}, o=L-2 R_{T}, p=L-R_{T}, k=2 R_{T}$ $-g$, and $g=L-x$.

The displacement of a point at the free end of flexure is given by the partial derivative of (2) with respect to the force applied as

$$
\begin{aligned}
& u_{i}=\frac{\partial U}{\partial F_{i}}, \\
& \theta_{i}=\frac{\partial U}{\partial M_{i}} .
\end{aligned}
$$

The compliance/stiffness can be calculated as the ratio of the displacement and the applied force.

A double-hinged straight flexure parameters were modified to obtain tapered flexures. For variation in heights at

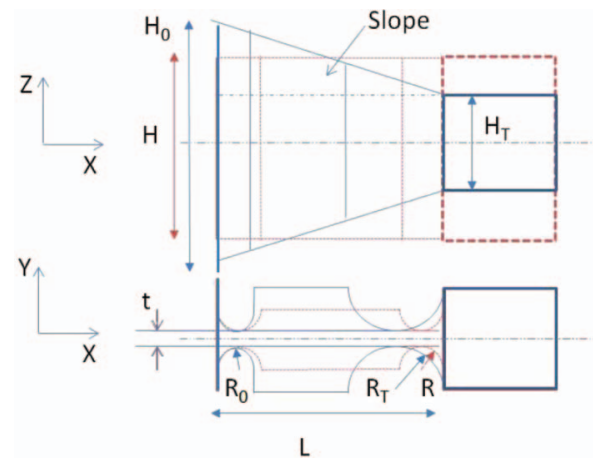

FIG. 2. Straight flexure (in red) and tapered flexure (in blue).

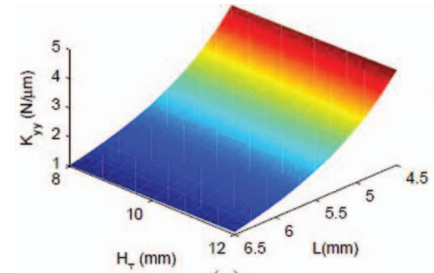

(a)

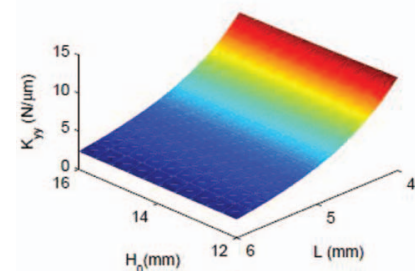

(c)

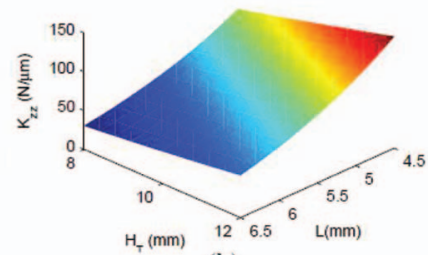

(b)

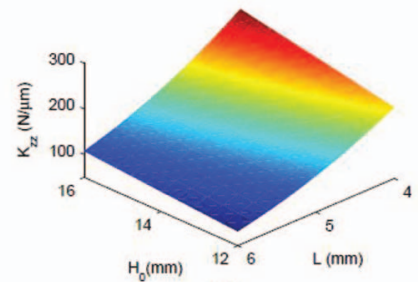

(d)
FIG. 3. Parametric variation of bending and out-of-plane stiffness of tapered flexures: (a) $K_{y y}$ vs $H_{T}$ vs $L$, (b) $K_{z z}$ vs $H_{T}$ vs $L$, (c) $K_{y y}$ vs $H_{0}$ vs $L$, and (d) $K_{z z}$ vs $H_{0}$ vs $L$.

tapered and fixed ends, the stiffness values of $K_{y y}$ and $K_{z z}$ were plotted as shown in Figs. 3. Figs. 3(a) and 3(c) show that with variation in height at tapered and fixed ends and with changed radii of respective hinges, the stiffness $K_{y y}$ remains unchanged. However, the out-of-plane stiffness decreases with decrease in $H_{T}$. It is inferred that the loss in the out-of-plane stiffness by the decrease in $H_{T}$ can be compensated with the increase in $H_{0}$. Also with the decrease in $H_{T}$, the height of the central stage is reduced, see Fig. 2. Thus, the mass of the stage is reduced by the same ratio as height. Let $\beta=H / H_{T}=M_{s} / M_{s}^{*}$, where $H$ and $M_{s}$ are the height and mass of the stage with straight flexures, $H_{T}$ and $M_{s}^{*}$ are the height and mass of the stage with tapered flexures, and $\beta$ is the ratio of heights or masses. The natural frequency will increase by a ratio of $\sqrt{\beta}$.

\section{DESIGN COMPARISON}

Designs of fast serial-kinematic nanopositioners with straight and tapered flexures are compared in this section. The aim is to achieve a high natural frequency along with an acceptable travel range. For the design case where straight flexures are used, only three parameters, i.e., radius $\mathrm{R}$, thickness $t$, and length $\mathrm{L}$, are evaluated for achieving the required stiffness. Nominal values for these parameters were adopted from the analysis presented by Wadikhaye et al., ${ }^{10}$ and iterated using finite-element-analysis to achieve the first natural frequency of $14 \mathrm{kHz}$ with an achievable travel range of $8.5 \mu \mathrm{m}$. The final values of the three parameters are $\mathrm{R}=0.6 \mathrm{~mm}, \mathrm{t}=0.5 \mathrm{~mm}$, and $\mathrm{L}=5 \mathrm{~mm}$. The height of flexures is equal to the height of the central stage, $\mathrm{H}=12.8 \mathrm{~mm}$. The lateral dimensions of the stage are $12 \mathrm{~mm} \times 12 \mathrm{~mm}$. The bending stiffness $K_{y y}$ was calculated to be $5.6 \mathrm{~N} / \mu \mathrm{m}$ per flexure and the out-of-plane stiffness $K_{z z}$ was calculated as $32 \mathrm{~N} / \mu \mathrm{m}$. The second mode which is a twisting mode was observed at $34 \mathrm{kHz}$ and the out-of-plane mode was observed at $38 \mathrm{kHz}$. 


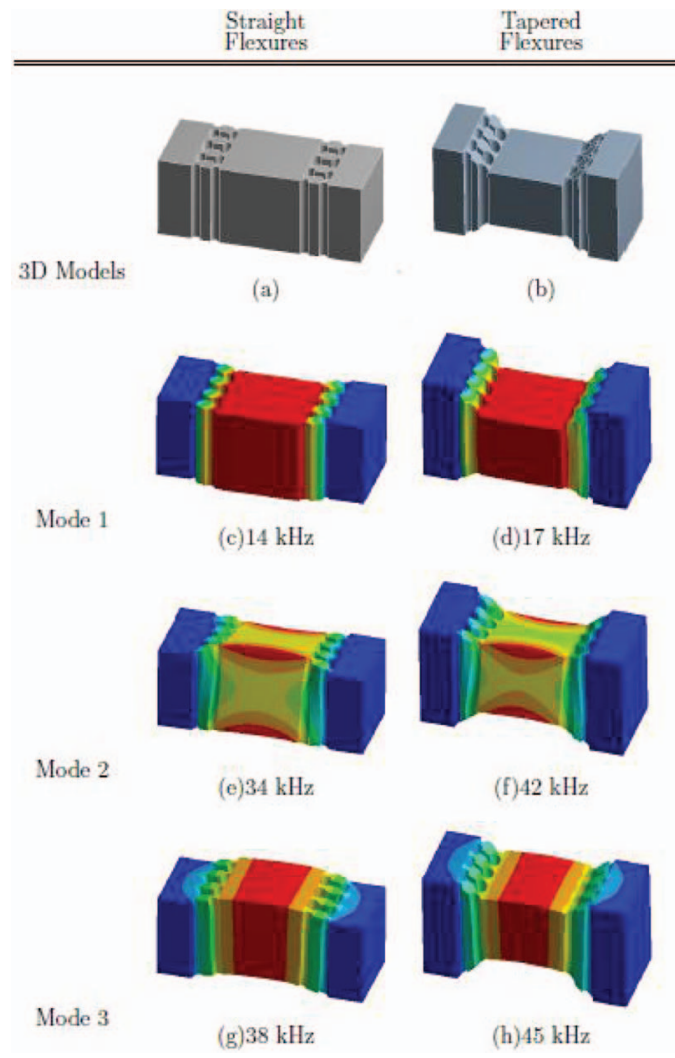

FIG. 4. Comparison of designs for fast stage of a serial-kinematic nanopositioner with (a), (c), (e), (g) straight flexures and (b), (d), (f), (h) tapered flexures.

The design of the nanopositioning stage using tapered flexures was carried out by modifying the heights and the radii of hinges of the straight flexures based on the radius correction presented in Sec. II. The thickness $\mathrm{t}=0.5 \mathrm{~mm}$ and length $\mathrm{L}=5 \mathrm{~mm}$, which are identical to the straight flexures designed above. The values of heights $\left(H_{0}\right.$ and $\left.H_{T}\right)$ are chosen from Figs. 3(a) to 3(d), such that the bending stiffness and out-of-plane stiffness are the same as that of straight flexures for a fair comparison. The radii of hinges are changed as per the ratio of heights given in (1). Finite-element-analysis showed that the first natural frequency was at $17 \mathrm{kHz}$ with the achievable travel range of $8.5 \mu \mathrm{m}$. The height $H_{0}$ is $16 \mathrm{~mm}$ and the height $H_{T}$ is $8 \mathrm{~mm}$. The height of the central stage is reduced to $2 / 3$ of the original stage, thus increasing the natural frequency by $25 \%$. The lateral dimensions of the central stage are the same. The bending stiffness was calculated to be $5.7 \mathrm{~N} / \mu \mathrm{m}$ and the out-of-plane stiffness was calculated to be $33.78 \mathrm{~N} / \mu \mathrm{m}$. Mass reduction also increases resonance frequency of the second and third modes as compared to the straight flexure design. The twisting mode was observed at $40 \mathrm{kHz}$ while the out-of-plane mode was observed at $43 \mathrm{kHz}$ (see Fig. 4). It can be inferred that with tapered flexures the first mode is well separated from the second and third modes as compared to the straight flexures.

\section{CROSS-COUPLING MEASUREMENTS}

Cross-coupling is a key design specification for a nanopositioner which affects its positioning accuracy. In the nanopositioners with a serial-kinematic configuration, this attribute is generally neglected because of the difficulty in measuring it. Generally, while deciding a control strategy for such nanopositioners cross-coupling is considered negligible, and thus, amounts to unmodeled dynamics. However, it has been shown that such effects could become significant in cases such as high-speed AFM. ${ }^{28,29}$ In the serial-kinematic configuration, these effects can be complex since stages with dissimilar dynamics are nested together. Therefore, there is a need for better understanding of cross-coupling effects for such nanopositioners.

The intricate arrangement of encompassed stages requires special arrangements for mounting the sensors. Fig. 5 shows different configuration in which the sensors can be mounted in a serial-kinematic configuration. For instance, for measuring the motion of the sample caused by $\mathrm{X}$-stage, the sensor needs to go through the Y-stage and sense at the walls of the X-stage. There can be two mounting positions possible for securing the sensor. The sensor can be mounted on the Ystage (Sensor X Position 1$),{ }^{8}$ or on the stationary frame (Sensor X Position 2) as is the case for most of the nanopositioners presented in the literature. ${ }^{10,30}$ In the first case, the crosscoupling from the Y-stage is neglected as the sensor moves with the Y-stage. In this arrangement, the sensor weight is added to the Y-stage which makes the stage asymmetric and causes a considerable change in the dynamics of the stage. The motion of the X-stage actuation and/or Y-stage crosscoupling in $x$ direction can be measured in the second configuration. As the sensor is secured to a static frame, the measurements are relative to the stationary reference.

To measure the displacement of the sample caused by Ystage, the sensor cannot be mounted on the Y-stage. In this case, the mounting position is fixed but the displacement can be sensed at two possible targets. First, the sensor can sense the displacement of the Y-stage itself at the Sensor Y Sensing Position 1 or the sensor can go through the Y-stage and sense motion of the $\mathrm{X}$-stage at the Sensor Y Sensing Position 2; see Fig. 5. In the first case, the sensor only sees the motion of the Y-stage and the cross-coupling from the X-stage is neglected. This configuration has been used in all the serialkinematic nanopositioners reported in the literature. ${ }^{6,8,10,30}$

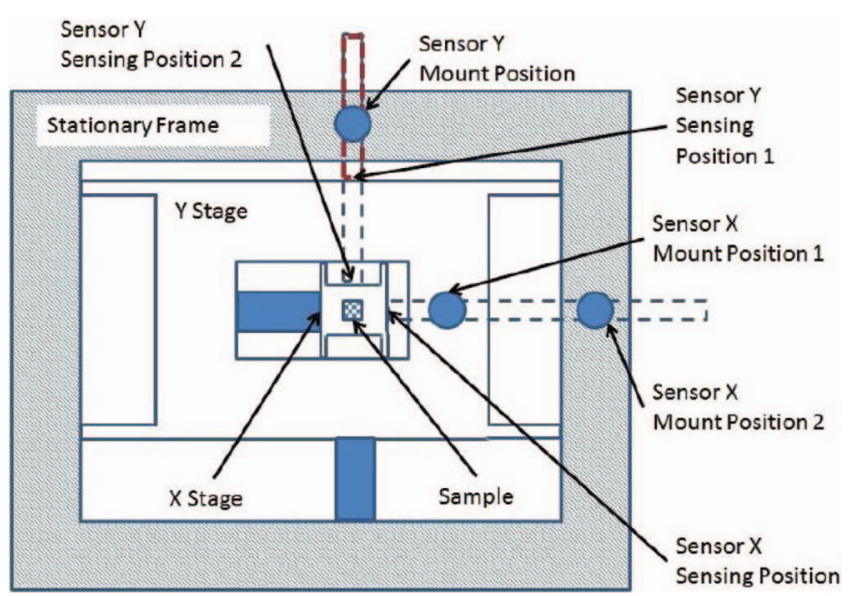

FIG. 5. Schematic of possible sensor positions for a nanopositioner with serial-kinematic configuration. 
Alternatively with the second case, the sensor measures any cross-coupling which may arise due to improper installation of the piezoelectric actuator of the X-stage or due to manufacturing imperfections. Thus, it can be said that if the sensors $\mathrm{X}$ and $\mathrm{Y}$ are mounted on the fixed frame and the displacements are measured at the X-stage where the sample is mounted, exact motion of the sample can be measured. The positioning accuracy can then be improved by efficient control techniques.

\section{HIGH-SPEED NANOPOSITIONER}

Based on the ideas discussed above a flexure-guided $\mathrm{XYZ}$ nanopositioner with a serial-kinematic configuration is proposed in this paper. The device has a fast (X) stage and a slow (Y) stage which makes it particularly suitable for raster scanning. It is a monolithic design with the $\mathrm{X}$-stage encompassed in the Y-stage. Piezoelectric actuators are used to drive each stage of the nanopositioner. The Z-stage is built by placing a small piezoelectric stack actuator in the cavity provided in the X-stage. A plate flexure is used to provide stiffness resulting in dominant mode along the actuation direction. The $\mathrm{X}$ axis is equipped with tapered flexures to support the stage while the $\mathrm{Y}$ axis is built with straight flexures. The nanopositioner is fabricated using wire-electrical-discharge-machining (WEDM) technique to achieve a high level of accuracy and tolerance. Aluminium-7075 with Young's Modulus of $72 \mathrm{GPa}$ is used which provides a good strength to weight $(E / \rho)$ ratio. Piezoelectric stack actuators (Noliac SCMAP07) are used to drive the stage along $x$ and $y$ directions. These actuators have dimensions of $5 \times 5 \times 10 \mathrm{~mm}$, having a stroke of $11.8 \mu \mathrm{m}$ at maximum drive voltage of $200 \mathrm{~V}$ and with a capacitance of $380 \mathrm{nF}$. The $\mathrm{Z}$ axis piezoelectric stack actuator is NAC2012 having dimensions of $3 \times 3 \times 2 \mathrm{~mm}$ with a stroke of $3 \mu \mathrm{m}$ at maximum drive voltage of $200 \mathrm{~V}$ and have a capacitance of 65 nF. Piezo Drive PDL 200 low noise amplifiers were used, one for each stage, to drive the piezoelectric actuators. Flexures are used as a preload for the piezoelectric actuators. A mechanism is designed for preloading which uses a screw and nut arrangement to apply tension that deforms the flexures elastically. As a result of this deformation, the stage gets displaced, such that the actuator can be placed in the required position. The mechanism makes the preloading elegant and as easy as a flick of the wrist which otherwise require dead weights to apply forces up to few hundred Newtons. Also, flexure assisted preloading dispenses the use of arrangements in which actuators are preloaded by pushing an end cap with the help of screw and ball arrangement. ${ }^{9}$

In the current design, the $\mathrm{X}$ and $\mathrm{Y}$ displacements are measured on the perpendicular faces of the $\mathrm{X}$-stage on which the sample is mounted. ADE technologies 8810 with 5000 series active capacitive probes, are used for measuring the displacement. The probes are mounted on the frame and not on the stages such that the displacement is with respect to the stationary reference. The probe passes through the Y-stage to reach the target area. A clearance of 20 times the stroke of Y-stage is given between the probe and Y-stage. A metal shim was wrapped around the sensor area that passes though the frame to maintain the clearance between the sensor and the

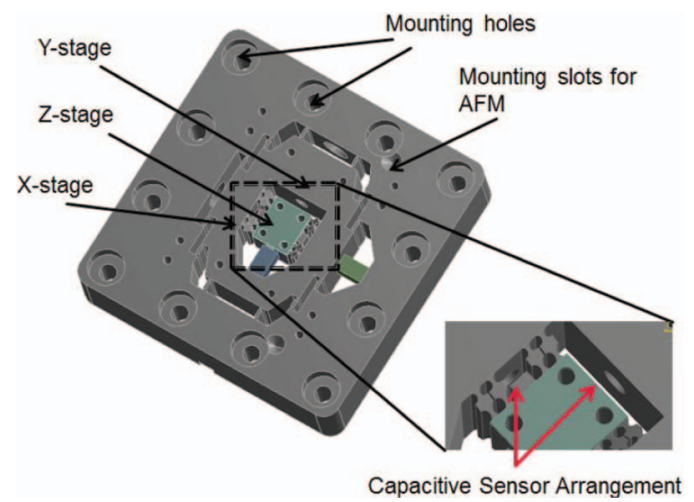

FIG. 6. High-speed XYZ nanopositioner design with the sensor arrangement.

Y-stage. The arrangement for measuring exact cross-coupling as seen by the sample is shown in Fig. 6 .

The analytical procedure described in Sec. II is an important tool for flexure design that can guide to achieve the specific requirements of complex systems like nanopositioners. The requirement in this case is to achieve a first resonance frequency greater than $20 \mathrm{kHz}$ with a travel range of $10 \mu \mathrm{m}$ in $x$ and $y$ directions. Initially, the stiffness calculations were carried out for flexures with straight geometry to obtain a stiffness of $10 \mathrm{~N} / \mu \mathrm{m}$ giving the estimate of the natural frequency of the X-stage around $18 \mathrm{kHz}$. The flexure geometry was then modified to tapered flexures by applying radius correction to obtain an identical stiffness. The estimated natural frequency with tapered flexures was calculated to be $25 \mathrm{kHz}$. The natural frequency for the Y-stage along the actuation direction was estimated to be $6 \mathrm{kHz}$. However, the natural frequency estimation using this analytical technique is based on single degree-of-freedom mass-spring system. A better picture of the system's dynamic behavior can be obtained from the modal analysis using Finite Element Analysis (FEA).

Modal analysis was carried out on the 3D model using ANSYS ${ }^{\circledR}$. The finite element model was established using 20-node SOLID186 element with refined mesh in the vicinity of flexure hinges and course mesh size elsewhere. Displacement constraints are assigned at the surfaces of the mounting holes to fix the nanopositioner in space. The first mode along the $\mathrm{Y}$ axis is seen at $6 \mathrm{kHz}$ while that along the $\mathrm{X}$ axis is observed at $23 \mathrm{kHz}$. The mode along the $\mathrm{Z}$ axis was seen at $155 \mathrm{kHz}$. Results of the FEA simulations of the nanopositioner are illustrated in Fig. 7.

\section{CHARACTERIZATION AND CONTROL}

The fabricated nanopositioner is mounted on a vibration isolation table and tested to determine its various system characteristics, such as positioning range, cross-coupling, hysteresis, and dynamic response: see Fig. 8. The nanopositioner is a three-input three-output system.

To evaluate the range along each axis and the crosscoupling between various axes of the nanopositioner, a $100 \mathrm{~Hz}$ sine wave of $200 \mathrm{~V}$ was applied to each of the piezoelectric stack actuators. The maximum displacements along $\mathrm{X}, \mathrm{Y}$, and $\mathrm{Z}$ axes were measured to be $8.5,8.5$, and $3 \mu \mathrm{m}$. 

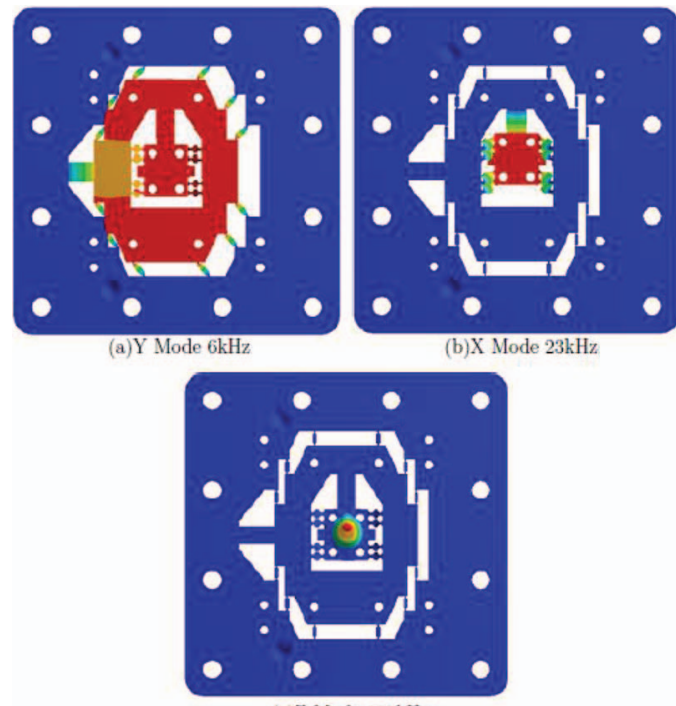

(c) Z Mode $155 \mathrm{kHz}$

FIG. 7. Finite element analysis results showing the first modes for (b) X-, (a) Y-, and (c) Z-stages.

These are represented as the diagonal elements of the subfigures matrix in the Fig. 9. When the $\mathrm{X}$ axis is actuated to achieve the full range by a $200 \mathrm{~V}$ sine wave, the crosscoupling motions induced in $\mathrm{Y}$ and $\mathrm{Z}$ axes are $1.3 \%(-37.7$ $\mathrm{dB})$ and $0.7 \%(-43 \mathrm{~dB})$ of the $\mathrm{X}$ axis displacement, respectively. When the $\mathrm{Y}$ axis is actuated, the induced crosscoupling along $\mathrm{X}$ and $\mathrm{Z}$ axes are $1.1 \%(-39.3 \mathrm{~dB})$ and $0.3 \%$ $(-43.5 \mathrm{~dB})$, respectively. Finally, when $\mathrm{Z}$ axis is actuated the induced $\mathrm{X}$ and $\mathrm{Y}$ cross-couplings are $0.17 \%(-55 \mathrm{~dB})$ and $0.17 \%(-55 \mathrm{~dB})$. To study the effects due to hysteresis, a $10 \mathrm{~Hz}$ sine wave of $200 \mathrm{~V}$ is applied along each axis. The response of the system is measured and plotted against the input signals to give the hysteresis loops as presented in Fig. 10. To quantify the hysteresis the maximum width of the hysteresis loop is taken as the parameter expressed as the per-

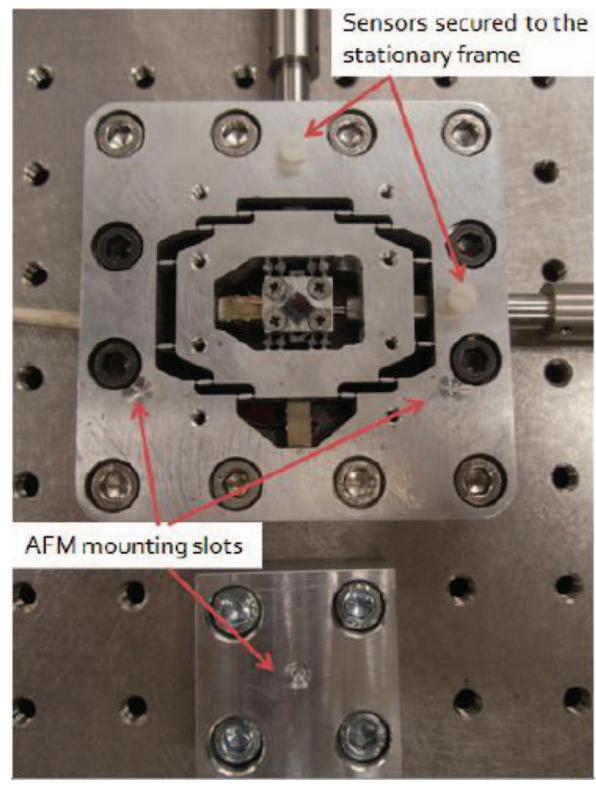

FIG. 8. Prototype nanopositioner. cent of the full range along the respective axis. Along $\mathrm{X}$ axis the hysteresis measured is $20 \%$, along $\mathrm{Y}$ and $\mathrm{Z}$ axes it is measured as $17.6 \%$ and $17.1 \%$, respectively. To identify the linear models of these transfer functions, a HP35670A dual channel spectrum analyzer was used to generate a swept sine input of $50 \mathrm{mVpk}$ with frequency range of $1 \mathrm{kHz}-50 \mathrm{kHz}$. These signals were applied to drive the piezoelectric stack actuators along the $\mathrm{X}, \mathrm{Y}$, and $\mathrm{Z}$ axes and the $\mathrm{X}$ and $\mathrm{Y}$ axes capacitive sensor responses $\left(v_{x}\right.$ and $v_{y}$ ) were recorded. A Polytec Laser Scanning Vibrometer, PSV 400 was used to record the response along the $\mathrm{Z}$ axis $\left(v_{z}\right)$. The measured open loop frequency responses are presented in Fig. 11. The first dominant mode for $\mathrm{X}, \mathrm{Y}$, and $\mathrm{Z}$ axes are $4.5 \mathrm{kHz}, 20 \mathrm{kHz}$, and $68 \mathrm{kHz}$, respectively. To the authors' knowledge, transfer function $G_{x y}$ has not been directly estimated for any serial-kinematic configuration in the past. It shows that, there is an appreciable cross-coupling of around $-20 \mathrm{~dB}$ from X-stage to Y-stage around the Y-stage resonance. The transfer function $G_{y x}$ does not need special arrangement for measuring it, but, has never been reported in literature. Again, in this case, there is a crosscoupling greater than $-20 \mathrm{~dB}$ from $\mathrm{Y}$-stage to X-stage. It can be inferred from the frequency response that $\mathrm{x}$ to $\mathrm{y}$ and $\mathrm{y}$ to $\mathrm{x}$ cross-coupling is significant within the proposed bandwidth of the nanopositioner.

\section{A. Feedforward control}

In this section, we present a feedforward control technique to mitigate the effects of dynamics and nonlinearities in the nanopositioning system. The goal of feedforward technique is to augment the plant and find the input $u$ such that when it is applied to the plant with known dynamics $G$, it produces the desired output $y_{d} \cdot 1,20,31$ In other words, it is desirable to acquire a $u$ such that

$$
Y_{d}(i \omega)=G(i \omega) U(i \omega)
$$

where $U(i \omega)$ and $Y_{d}(i \omega)$ are Fourier transforms of $u$ and the desired trajectory $y_{d}$, respectively. Generally, the feedforward approach uses the inverse of mathematical model of the system's linear dynamics $\mathcal{G}^{-1}$ to mitigate the dynamics of the system. This system is then augmented with the hysteresis inverse $\mathcal{H}^{-1}$ to give the inverse of the entire system as $\mathcal{H}^{-1}\left[\mathcal{G}^{-1}\left(y_{d}\right)\right]$.

Inversion of the system dynamics is rudimentary for feedforward control, the challenge is to model the hysteresis effects. Several modeling techniques have been proposed and used in the literature such as Preisach model ${ }^{15}$ and PrandtleIshlinshkii model. ${ }^{22}$ However, such approaches are demanding in terms of modeling complexity and parameter sensitivity. The phaser approach, as presented in Ref. 24 avoids the need for an accurate model. In this approach, the hysteresis is represented as a linear phase lag that can be compensated for by using a phase lead transfer function in series with the plant. In this paper, input signals are shaped using the linear dynamic response and the phaser approximation of hysteresis at the harmonics present in the reference signals. The desired capacitive sensor output should be a smooth triangular signal for triangular input which in Fourier series form can be 

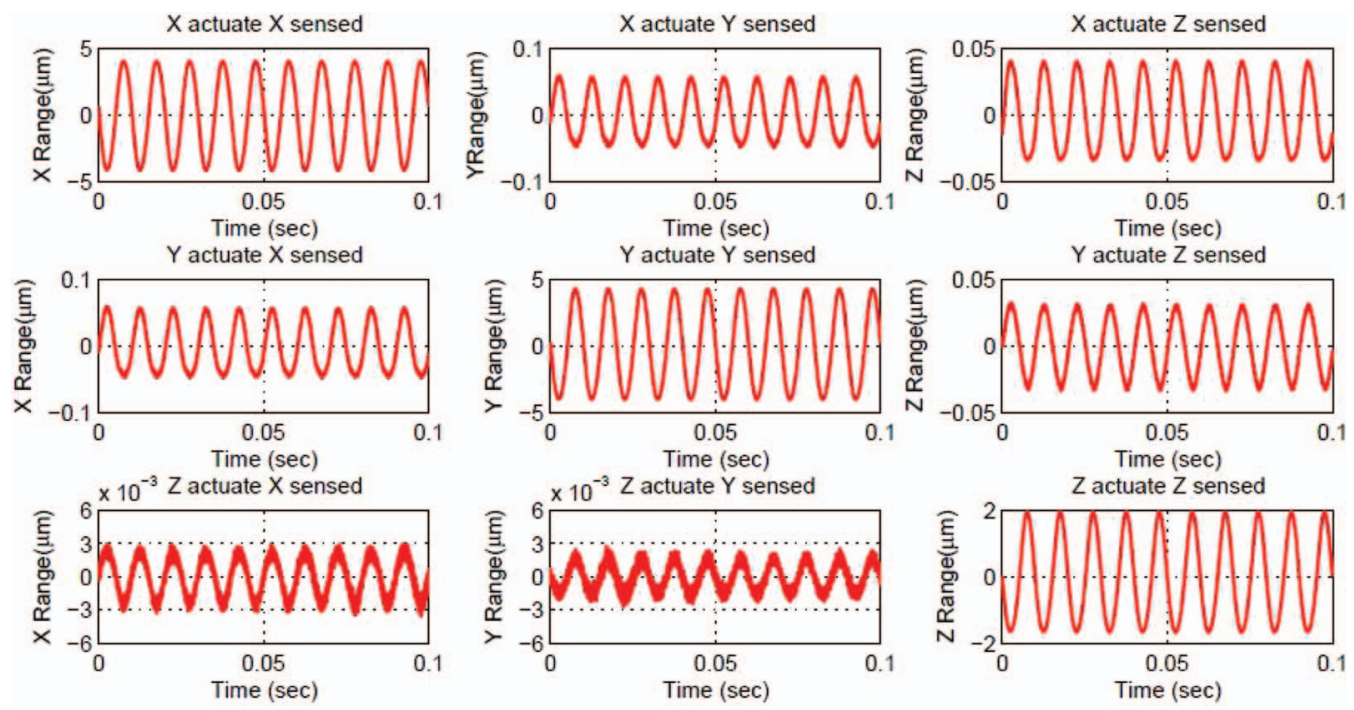

FIG. 9. Measured displacements and cross-coupling motions of the XYZ nanopositioner.

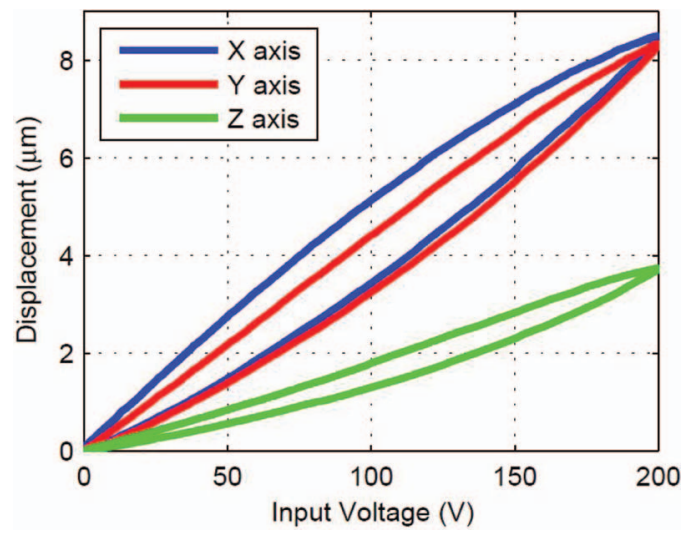

FIG. 10. Plots showing the hysteresis loop obtained by plotting the input voltage versus the output displacement for X-, Y-, and Z-stages. written as

$$
r(t)=\sum_{k=1}^{\infty} A_{k} \sin \left(\omega_{k} t\right)
$$

where $A_{k}=\frac{8}{\pi^{2} k^{2}} \sin \left(\frac{\pi k}{2}\right)$ and $\omega_{k}=2 \pi k f$, with $f$ being the fundamental frequency of the triangular waveform. To eliminate the hysteretic effects in the output, instead of using a triangular wave, inputs to the actuator are shaped such that the output $y_{d}(t)$ is a triangular signal. The amplitude and phase of harmonics are rectified as per the system characterization such that a pseudo triangular waveform is obtained as

$$
u(t)=\sum_{k=1}^{\infty} \frac{A_{k}}{\left|G_{x x}\left(i \omega_{k}\right)\right|} \sin \left(\omega_{k} t-\phi_{k}\right),
$$

where $k=1,2, \ldots$
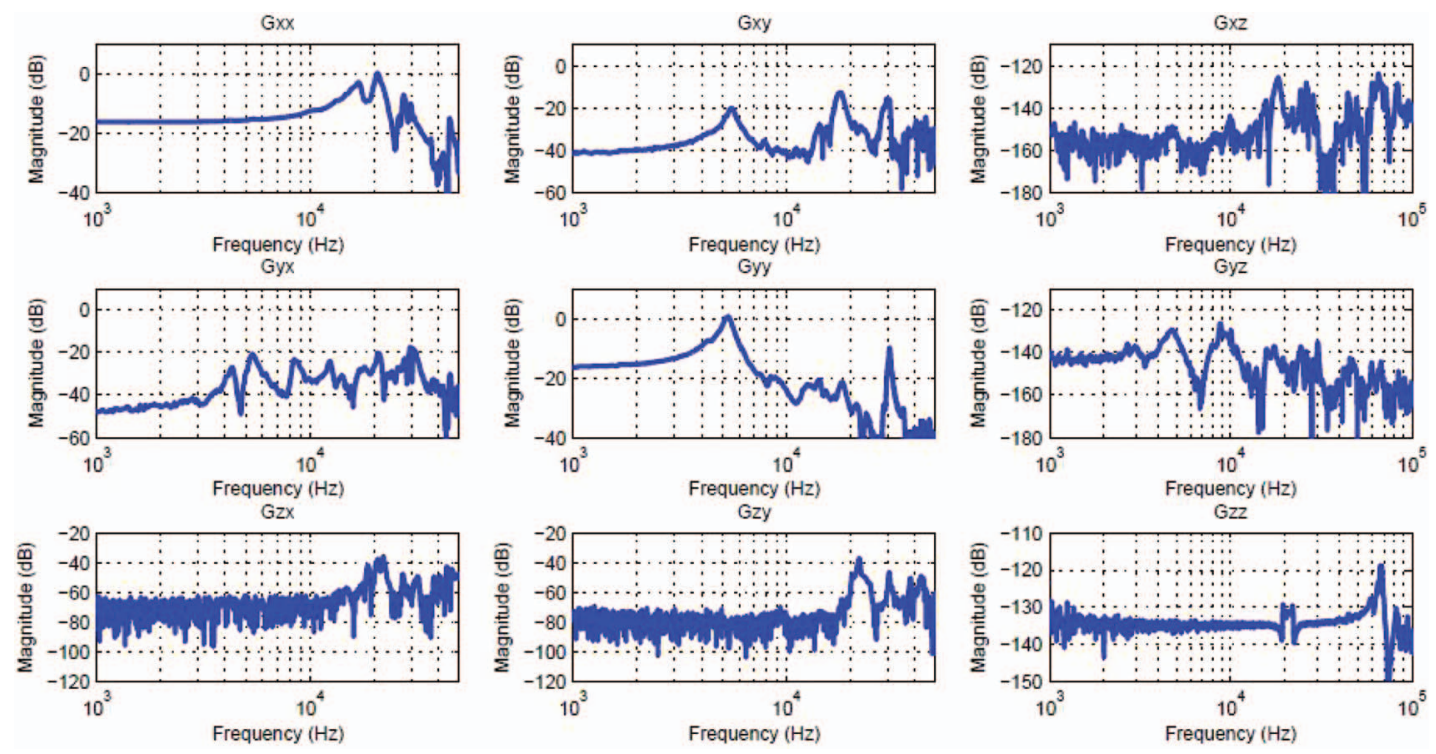

FIG. 11. Measured frequency responses of the three-input-three-output nanopositioner. 


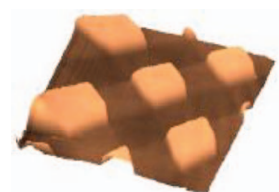

(a) $160 \mathrm{~Hz}$ OL

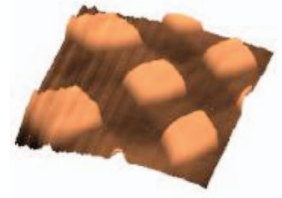

(c) $320 \mathrm{~Hz}$ OL

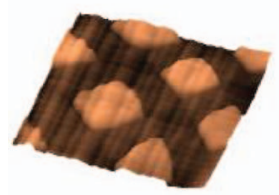

(e) $640 \mathrm{~Hz} \mathrm{OL}$

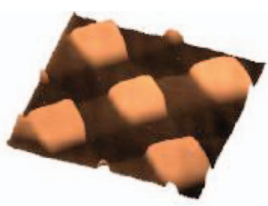

(b) $160 \mathrm{~Hz} \mathrm{FF}$

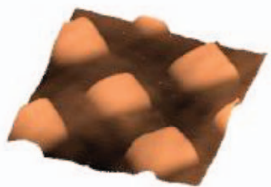

(d) $320 \mathrm{~Hz}$ FF

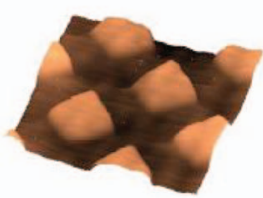

(f) $640 \mathrm{~Hz} \mathrm{FF}$
FIG. 12. Scanned images of a scan grating at $160 \mathrm{~Hz}, 320 \mathrm{~Hz}$, and $640 \mathrm{~Hz}$. Figures (a), (c), and (e) are open loop scans and figures (b), (d), and (f) are scans with feedforward control for the said scan speeds, respectively.

Here, phase angle $\phi_{k}$ is a combination of a linear component $\angle G_{x x}\left(i \omega_{k}\right)$ and a hysteretic component $\Lambda_{k}$. The linear component is obtained from the small amplitude frequency response of the nanopositioner, while, the hysteretic component is approximated as a phase shift. ${ }^{24} \mathrm{~A}$ LabVIEW virtual instrument was built for generating these shaped signals from the harmonic content of the reference triangular signals and the system response.

\section{IMAGING PERFORMANCE}

The nanopositioner was integrated with a commercially available NanoSurf easyScan 2 AFM. The inbuilt XYnanopositioner of the AFM was made redundant. It should be mentioned that the maximum achievable scanning speed of the commercial AFM using its inbuilt nanopositioner is 10 $\mathrm{Hz}$. The AFM was set to operate in the constant height mode due to limitations on achievable controller gains along the $\mathrm{Z}$ axis. A high stiffness tapping mode AFM cantilever (Tap 190G) with resonance of $190 \mathrm{kHz}$ and spring constant of $48 \mathrm{~N} / \mathrm{m}$, was used instead of the conventional $13 \mathrm{kHz}$, ContAl contact mode cantilever with a spring constant of $0.2 \mathrm{~N} / \mathrm{m}$. The high stiffness cantilever was necessary since the resonance of the conventional cantilever were within the bandwidth of the proposed nanopositioner. The set point for landing this high stiffness cantilever was set to a lower value, proportional to the stiffness of the cantilever. This was essential to avoid high forces being applied to the sample and to avoid tip wear. The $\mathrm{x}$ and $y$ signals of the raster scanning trajectory were generated using a custom user interface developed for controlling the parameters. This user interface was developed in a LabView programming environment which integrates the National Instruments data acquisition card using NI-DAQmx drivers. A NI PCI-6115 high-speed simultaneous sampling data acquisition card, installed in a NI PXIe 8110 chassis was used to achieve input-output sampling rates up to $2 \mathrm{MS} / \mathrm{s}$.

To evaluate the imaging performance of the proposed nanopositioner, a MikroMasch TFQ1 calibration grating with

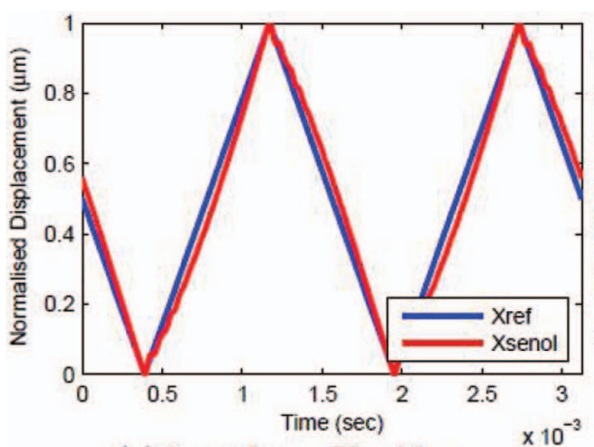

(a)Open Loop Tracking

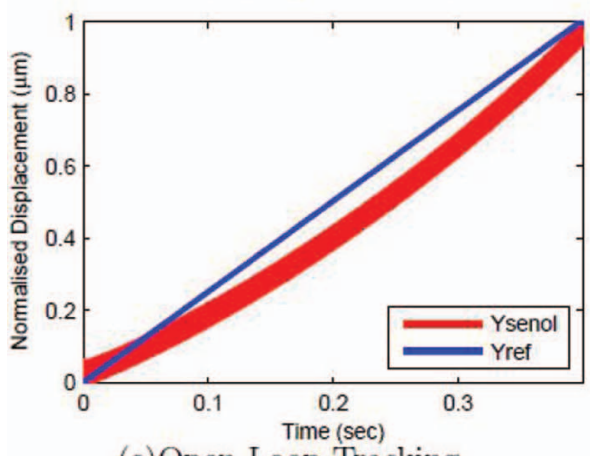

(c) Open Loop Tracking

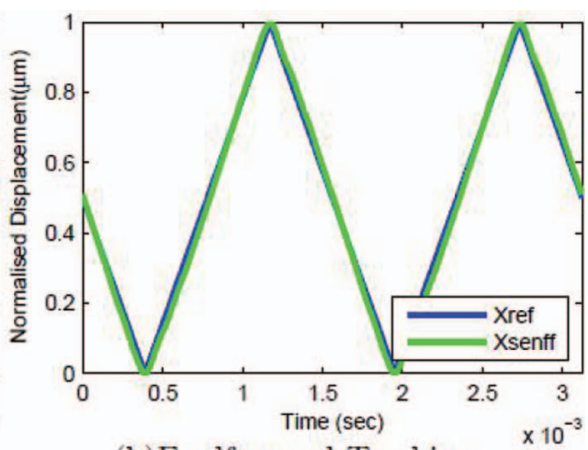

(b)Feedforward Tracking

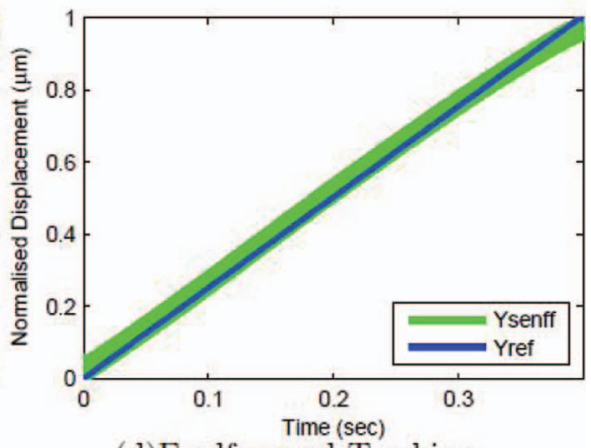

(d)Feedforward Tracking

FIG. 13. Measured tracking results: (a) and (b) Tracking performance of the nanopositioner along X axis at $640 \mathrm{~Hz}$. (c) and (d) Tracking performance of the nanopositioner along $\mathrm{Y}$ axis. 
$3 \mu \mathrm{m}$ period, $1.5 \mu \mathrm{m}$ square side, and $20 \mathrm{~nm}$ height was scanned. An area of $7 \mu \mathrm{m} \times 7 \mu \mathrm{m}$ was scanned at a resolution of $256 \times 256$ pixels. Images were captured in the open loop and with the feedforward control at scanning speeds of $160 \mathrm{~Hz}, 320 \mathrm{~Hz}$, and $640 \mathrm{~Hz}$. Higher scanning rates were not attempted to avoid wear of the tip due to scan induced vibrations. The scanned images are created by plotting the cantilever deflection against the desired lateral position as shown in Fig. 12. For open loop scan, the scan induced vibrations can be clearly seen at $320 \mathrm{~Hz}$ and $640 \mathrm{~Hz}$. Moreover, another artifact which can be distinctively seen on all open loop images is the bent alignment of the features. These artifacts are due to the presence of hysteresis in the operation of piezoelectric actuators.

The images scanned with feedforward control show that the scan induced oscillations are almost non-existent at 160 and $320 \mathrm{~Hz}$. Image quality at $640 \mathrm{~Hz}$ is comparatively lower than that of $160 \mathrm{~Hz}$ and $320 \mathrm{~Hz}$. This may be due to the fact that the higher harmonics of $640 \mathrm{~Hz}$ are significantly closer to the resonance of the stage. Also, in contrast to the open loop scans, the hysteresis effects were significantly compensated. The tracking performance at $640 \mathrm{~Hz}$ is shown in Fig. 13, which depicts that the oscillation in the triangular signals in open loop are significantly reduced with the feedforward control. Also the hysteresis effects in X as well as the Y-stage are minimized considerably. The RMS tracking error for X (triangular) signals at $640 \mathrm{~Hz}$ for open loop was calculated to be $4 \%$ of the full range while that with the feedforward control it was $1 \%$. Similarly, the Y (ramp) signals for $640 \mathrm{~Hz}$ scan in open loop have RMS tracking error of $6 \%$ while with the feedforward it was $2.5 \%$. However, the error value along $\mathrm{Y}$ axis is high mostly due to the dynamic interaction from X-stage. This error can be further reduced if the cross-coupling from the $\mathrm{X}$ axis is controlled.

\section{CONCLUSION}

Design of a serial-kinematic nanopositioner with tapered flexures is presented in this paper. Parametric evaluation shows that the mass of the nanopositioning stage can be reduced using tapered flexures and hence enhance the natural frequency of the stage. Sensor configuration for serial-kinematic design is also studied. The best configuration which gives an exact measurement of displacement and cross-coupling as seen by sample is suggested and used in the design. A high-speed XYZ nanopositioner is presented based on tapered flexures and best sensor configuration is presented. The first resonant mode for X-, Y-, and Z-stages are reported as $20 \mathrm{kHz}, 4.5 \mathrm{kHz}$, and $64 \mathrm{kHz}$. The measured range of the nanopositioner along $\mathrm{x}, \mathrm{y}$, and $\mathrm{z}$ axes are reported to be $8.5 \mu \mathrm{m}, 8.5 \mu \mathrm{m}$, and $3 \mu \mathrm{m}$. Cross-coupling along the three axes, which is generally neglected in serial-kinematic nanopositioners, is also characterized and reported. A feedforward control based on the phaser approach to model the hysteresis is also presented. It is observed that with the proposed controller the dynamic and hysteresis effects are mitigated considerably. However, the cross-coupling effects from $\mathrm{X}$ to $\mathrm{Y}$ axes are not suppressed. In future work, authors plan to implement a multivariable controller to overcome the crosscoupling issue.

\section{ACKNOWLEDGMENTS}

The reported research was performed at Laboratory for Dynamics and Control of Nanosystems (LDCN) at the University of Newcastle, Callaghan, N.S.W., Australia. This work was supported by the Australian Research Council (ARC) and the University of Newcastle.

${ }^{1}$ S. Devasia, E. Eleftheriou, and S. Moheimani, "A survey of control issues in nanopositioning," IEEE Trans. Control Syst. Technol. 15(5), 802-823 (2007).

${ }^{2}$ Y. K. Yong, S. O. R. Moheimani, B. J. Kenton, and K. K. Leang, "Invited review article: High-speed flexure-guided nanopositioning: Mechanical design and control issues," Rev. Sci. Instrum. 83(12), 121101 (2012).

${ }^{3}$ G. C. Loney, "High bandwidth steering mirror," U.S. patent 5110195 (March 1991).

${ }^{4}$ H. Lan, Y. Ding, H. Liu, and B. Lu, "Review of the wafer stage for nanoimprint lithography," Microelectron. Eng. 84(4), 684-688 (2007).

${ }^{5}$ T. Ando, N. Kodera, E. Takai, D. Maruyama, K. Saito, and A. Toda, "A high-speed atomic force microscope for studying biological macromolecules," Proc. Natl. Acad. Sci. U.S.A. 98(22), 12468-12472 (2001)

${ }^{6}$ K. K. Leang and A. J. Fleming, "High-speed serial-kinematic SPM scanner: Design and drive considerations," Asian J. Control 11(2), 144-153 (2009).

${ }^{7}$ G. Schitter, K. Astrom, B. DeMartini, P. Thurner, K. Turner, and P. Hansma, "Design and modeling of a high-speed AFM-scanner," IEEE Trans. Control Syst. Technol. 15(5), 906-915 (2007).

${ }^{8}$ B. J. Kenton and K. K. Leang, "Design and control of a three-axis serialkinematic high-bandwidth nanopositioner," IEEE/ASME Trans. Mechatron. 17(2), 356-369 (2012).

${ }^{9}$ Y. K. Yong, B. Bhikkaji, and S. O. R. Moheimani, "Design, modeling and FPAA-based control of a high-speed atomic force microscope nanopositioner," IEEE/ASME Trans. Mechatron. 18(3), 1060-1071 (2013).

${ }^{10}$ S. P. Wadikhaye, Y. K. Yong, and S. O. R. Moheimani, "Design of a compact serial-kinematic scanner for high-speed atomic force microscopy: An analytical approach," Micro Nano Lett. 7(4), 309-313 (2012).

${ }^{11}$ G. Schitter, P. Menold, H. F. Knapp, F. Allgower, and A. Stemmer, "High performance feedback for fast scanning atomic force microscopes," Rev. Sci. Instrum. 72(8), 3320-3327 (2001).

${ }^{12}$ S. Salapaka, A. Sebastian, J. P. Cleveland, and M. V. Salapaka, "High bandwidth nano-positioner: A robust control approach," Rev. Sci. Instrum. 73(9), 3232-3241 (2002).

${ }^{13}$ Y. K. Yong, S. S. Aphale, and S. O. R. Moheimani, "Design, identification and control of a flexure-based XY stage for fast nanoscale positioning," IEEE Trans. Nanotechnol. 8(1), 46-54 (2009).

${ }^{14}$ T. Tuma, W. Haeberle, H. Rothuizen, J. Lygeros, A. Pantazi, and A. Sebastian, "A dual-stage nanopositioning approach to high-speed scanning probe microscopy," in Proceedings of the IEEE 51st Annual Conference on Decision and Control (CDC), December 2012 (IEEE, 2012), pp. 5079-5084.

${ }^{15}$ D. Croft, G. Shed, and S. Devasia, "Creep, hysteresis, and vibration compensation for piezoactuators: Atomic force microscopy application," J. Dyn. Syst., Meas., Control 123(1), 35-43 (2001).

${ }^{16} \mathrm{~S}$. Aphale, B. Bhikkaji, and S. Moheimani, "Minimizing scanning errors in piezoelectric stack-actuated nanopositioning platforms," IEEE Trans. Nanotechnol. 7(1), 79-90 (2008).

${ }^{17}$ B. Bhikkaji and S. Moheimani, "Integral resonant control of a piezoelectric tube actuator for fast nanoscale positioning," IEEE/ASME Trans. Mechatron. 13(5), 530-537 (2008).

${ }^{18}$ S. P. Wadikhaye, Y. K. Yong, B. Bhikkaji, and S. O. R. Moheimani, "Control of a piezoelectrically actuated high-speed serial-kinematic AFM nanopositioner," Smart Mater. Struct. 23(2), 025030 (2014).

${ }^{19}$ J. Dong, S. M. Salapaka, and P. M. Ferreira, "Robust control of a parallelkinematic nanopositioner," J. Dyn. Syst., Meas., Control 130(4), 041007 (2008).

${ }^{20}$ G. Clayton, S. Tien, K. K. Leang, Q. Zou, and S. Devasia, "A review of feedforward control approaches in nanopositioning for high-speed SPM," J. Dyn. Syst., Meas., Control 131(6), 061101 (2009).

${ }^{21} \mathrm{P}$. Ge and M. Jouaneh, "Generalized Preisach model for hysteresis nonlinearity of piezoceramic actuators,” Precis. Eng. 20(2), 99-111 (1997). 
${ }^{22}$ M. Rakotondrabe, C. Clevy, and P. Lutz, "Complete open loop control of hysteretic, creeped, and oscillating piezoelectric cantilevers," IEEE Trans. Autom. Sci. Eng. 7(3), 440-450 (2010).

${ }^{23} \mathrm{M}$. Goldfarb and N. Celanovic, "Modeling piezoelectric stack actuators for control of micromanipulation," IEEE Control Syst. 17(3), 69-79 (1997).

${ }^{24}$ J. Cruz-Hernandez and V. Hayward, "Phase control approach to hysteresis reduction," IEEE Trans. Control Syst. Technol. 9(1), 17-26 (2001).

${ }^{25}$ L. Meirovitch, Computational Methods in Structural Dynamics (Springer, 1980).

${ }^{26}$ S. P. Wadikhaye, Y. K. Yong, and S. O. R. Moheimani, "Nanopositioner design using tapered flexures: A parametric study," in Proceedings of the IEEE/ASME International Conference on Advanced Intelligent Mechatronics (AIM), July 2013 (IEEE, 2013), pp. 856-861.
${ }^{27}$ S. Timoshenko and J. Goodier, Theory of Elasticity, 3rd ed. (McGraw-Hill, 1970).

${ }^{28} \mathrm{O}$. El Rifai and K. Youcef-Toumi, "Coupling in piezoelectric tube scanners used in scanning probe microscopes," in Proceedings of the American Control Conference, 2001, Vol. 4, pp. 3251-3255.

${ }^{29}$ Y. K. Yong, K. Liu, and S. O. R. Moheimani, "Reducing cross-coupling in a compliant XY nanopositioning stage for fast and accurate raster scanning," IEEE Trans. Control Syst. Technol. 18(5), 1172-1179 (2010).

${ }^{30}$ T. Ando, T. Uchihashi, N. Kodera, D. Yamamoto, A. Miyagi, M. Taniguchi, and H. Yamashita, "High-speed AFM and nano-visualization of biomolecular processes," Pflügers Arch. Eur. J. Physiol. 456, 211-225 (2008).

${ }^{31}$ S. S. Aphale, S. Devasia, and S. O. R. Moheimani, "High-bandwidth control of a piezoelectric nanopositioning stage in the presence of plant uncertainties," Nanotechnology 19, 125503 (2008). 